\title{
FACTORES PARA LA PUESTA EN MARCHA Y EL ÉXITO DE MICROEMPRESAS ASOCIATIVAS CREADAS POR JÓVENES EGRESADOS UNIVERSITARIOS
}

\author{
POR \\ Jorge COQUE MARTÍNEZ², \\ Francisco DÍAZ BRETONES ${ }^{3}$ y \\ Nuria LÓPEZ MIELGO ${ }^{4}$
}

\section{RESUMEN}

El fomento del emprendimiento desde las universidades es una función académica cada vez más valorada. Sin embargo, existen muy pocos trabajos que aborden las barreras y los elementos facilitadores que encuentran los emprendedores universitarios. Este estudio analiza los motivos y otros factores que facilitan la puesta en marcha y el éxito de empresas colectivas creadas por universitarios poniendo especial énfasis en el papel desempeñado por las universidades. El análisis empírico consistió en el estudio de 13 empresas creadas por egresados de tres universidades españolas, mediante entrevistas en profundidad a sus socios. Los resultados muestran que el desarrollo profesional y valores personales como iniciativa, independencia, capacidad de adaptación y constancia son las motivaciones más señaladas por los universitarios para crear empresas con sus compañeros. Asimismo, la universidad aparece como un elemento de apoyo a través de asignaturas de creación de empresas, servicios como asesoría, publicidad o contactos y el conocimiento mutuo de futuros socios. Sin embargo, el

\footnotetext{
${ }^{1}$ Este trabajo fue desarrollado gracias a la financiación recibida por la Agencia Española de Cooperación Internacional para el Desarrollo (AECID) a través de su Programa de Cooperación Interuniversitaria e Investigación Científica entre España e Iberoamérica (Resolución de 14 de diciembre de 2007. Referencia A/7626/07).

${ }^{2}$ Jorge COQUE MARTÍNEZ: Universidad de Oviedo. Dirección de correo electrónico: coque@ uniovi.es

${ }^{3}$ Francisco DÍAZ BRETONES: Universidad de Granada. Dirección de correo electrónico: fdiazb@ugr.es

${ }^{4}$ Nuria LÓPEZ MIELGO: Universidad de Oviedo. Dirección de correo electrónico: nlopez@uniovi.es

REVESCO No 112 - Extraordinario en Homenaje al Profesor Alfonso Carlos MORALES

GUTIÉRREZ - ISSN: 1885-8031 - www.ucm.es/info/revesco

http://dx.doi.org/10.5209/rev_REVE.2013.v112.43063

Fecha de recepción: 06/12/2011

Fecha de aceptación: 07/02/2013
} 
análisis revela que aún queda mucho camino por recorrer en las universidades para lograr una promoción más eficaz.

Palabras clave: Emprendimiento colectivo, universidad, motivación, apoyo.

Claves Econlit: H810, I250, J540, L260

\title{
FACTORS FOR SETTING UP AND SUCCESS OF ASSOCIATIVE MICRO- BUSINESSES CREATED BY YOUNG UNIVERSITY GRADUATES
}

\begin{abstract}
The promotion of entrepreneurship from the universities is an increasingly valued academic function. However, few papers explore the barriers and facilitating elements that university entrepreneurs face. This study analyses the motivation and other factors that ease the setting up and success of collective firms created by university students, with special emphasis in the role played by universities. The empirical analysis is based on the study of 13 companies created by graduates from three Spanish universities, throug in depth interviews to its members. The results show that the professional development and personal values like initiative, independence, adjustment capacity and perseverance are the most pointed out motivations by university students to create a firm with their classmates. Likewise, university appears as an element of support with entrepreneurship courses, services as advising, advertising or contacts, and the mutual knowledge of future partners. Nevertheless, the analysis reveals that much work is still needed if universities want to offer an effective promotion.
\end{abstract}

Key words: Collective entrepreneurship, university, motivation, support.

\section{INTRODUCCIÓN}

La creación de empresas universitarias constituye una fuente de transferencia de conocimiento muy valorada en las últimas décadas debido a su capacidad para generar riqueza y crecimiento de la economía local. Por un lado, suelen situarse cerca de donde se crean, estimulando el crecimiento económico de la zona. Por otro, facilitan la incorporación de titulados universitarios favoreciendo el autoempleo y estimulando un mercado de 
conocimiento a través de los recursos humanos. Finalmente, facilitan una mejor valorización de los resultados de la investigación, puesto que la creación de una empresa universitaria constituye la vía más directa de comercializar la tecnología y el conocimiento (Rodeiro, Fernández, Otero y Rodríguez, 2010).

Por estas razones, la universidad debe ser un actor importante en la creación de empresas mediante estrategias dirigidas a fomentar el autoempleo y los comportamientos emprendedores entre sus egresados. Este aspecto cobra cada vez mayor importancia, de manera que en los nuevos planes de estudios aparece la formación en creación de empresas como una asignatura transversal en muchos de los títulos de grado.

Sin embargo, la labor de impulsar la concepción, gestación y nacimiento de empresas universitarias es una tarea que se encuentra sometida a serias dificultades. Estas empresas se forman en muchas ocasiones por varios titulados con el objetivo de explotar su conocimiento en el mercado para obtener un lucro y, además, mantener la propiedad y el control de la empresa creada, es decir, constituyen una empresa de economía social. En estos casos, existen riesgos de que la excesiva intervención de terceros aboque al posterior fracaso o a procesos de puesta en marcha plagados de dificultades (Morales 1996, 1998; Vargas, 1995). Asimismo, las acciones desarrolladas desde la universidad resultan en muchos casos insuficientes y descoordinadas.

Por todo ello, el objetivo de este trabajo se centra en analizar a las empresas de rasgos cooperativos creadas en la universidad. Más concretamente, se pretenden conocer los motivos que llevan a su creación así como los apoyos que encontraron en las primeras etapas de su ciclo de vida. Para ello, se ha realizado una investigación cuya exposición se estructura del siguiente modo. En primer lugar, se exponen los fundamentos teóricos relacionados con el proceso de generación de grupos emprendedores. Más adelante se aborda el papel de la universidad en el fomento del emprendimiento. Finalmente, se presenta un estudio empírico realizado con trece empresas de origen universitario de Asturias, Jaén y Granada, y algunas conclusiones y recomendaciones que se derivan de la investigación. 


\section{FUNDAMENTACIÓN TEÓRICA}

\subsection{La generación de grupos de emprendedores}

Toda empresa tiene un ciclo de vida que debería recorrer tres momentos clave: creación, mantenimiento y desarrollo, o infancia, juventud y madurez, según la analogía biológica (Veciana, 1999; Trusen, 1994). Abundantes estudios empíricos demuestran que todas las fases, incluida una previa (la gestación), están especialmente ligadas a las fuerzas y debilidades de los emprendedores (Entrialgo, Fernández y Vázquez, 2001; Rutherford y Oswald, 2000; Singh, Hybels y Hills, 2000; Raffa, Zollo y Caponi, 1996; Kelmar y Wingham, 1995). Por otro lado, el suceso disparador de la creación de una empresa suele encontrarse entre los siguientes factores (Veciana, 1999): necesidad de aprobar; necesidad de independencia; necesidad de desarrollo personal; para contribuir al bienestar de otros; para obtener lucro directo o indirecto; para perseguir un modelo; para huir de la marginación.

Estas consideraciones pueden extrapolarse a los fenómenos de emprendimiento colectivo que dan lugar a empresas de economía social (o, de modo más preciso, a empresas de participación, según García-Gutiérrez, 1991), que deben soportar problemas adicionales desde su fase de gestación, esto es, desde la creación del grupo de emprendedores (Tabla 1). Tales problemas deben solventarse combinando diversas medidas (Hunt, 1992; Cornforth y Thomas, 1990; Préfontaine y Léger, 1990): haciendo explícitos los objetivos, capacidades y recursos de cada uno de los posibles miembros de la futura empresa y dejando claro también cuáles son los valores que les unen, para analizar si realmente se trata de un grupo en términos de homogeneidad interna suficiente y decidir si se cuenta con una masa crítica humana necesaria y suficiente para dar el salto al mercado o, por el contrario, sobra o falta alguien; acotando las actividades a las que va a dedicarse la empresa, y comenzando a obtener los recursos necesarios para ponerla en marcha.

Tabla 1. La conformación de un grupo de emprendedores

\begin{tabular}{|c|c|}
\hline Objetivos & $\begin{array}{l}\text { - Conocimiento mutuo y creación del grupo (¿Quiénes vamos a ser?) } \\
\text { - Decisión sobre la actividad empresarial (¿A qué nos vamos a dedicar?) }\end{array}$ \\
\hline $\begin{array}{l}\text { Protagonismo en el colectivo de } \\
\text { emprendedores }\end{array}$ & - Líderes iniciales (empresario fundador) \\
\hline $\begin{array}{l}\text { Qué sucede en la organización } \\
\text { (fase del funcionamiento asambleario) }\end{array}$ & $\begin{array}{l}\text { - Participación generalizada y desorganizada, en ausencia de reglas claras. } \\
\text { - Límites de la organización difusos. } \\
\text { - Falta de concreción sobre ideas empresariales. }\end{array}$ \\
\hline Problemas a resolver & $\begin{array}{l}\text { - Identificación, selección y jerarquización de necesidades, separando las } \\
\text { individuales de las colectivas. } \\
\text { - Definición del primer grupo de emprendedores. } \\
\text { - Identificación de debilidades, amenazas, fuerzas y oportunidades. }\end{array}$ \\
\hline
\end{tabular}

REVESCO No 112 - Extraordinario en Homenaje al Profesor Alfonso Carlos MORALES GUTIÉRREZ - ISSN: 1885-8031 - www.ucm.es/info/revesco 


\begin{tabular}{|c|c|c|}
\hline \multicolumn{2}{|l|}{ Soluciones } & $\begin{array}{l}\text { - Análisis de relaciones previas entre futuros socios y de reglas de su } \\
\text { entorno. } \\
\text { - Búsqueda de cohesión grupal y toma de primeras decisiones colectivas } \\
\text { - Formación y asesoría externa que moderen la dinámica del grupo y } \\
\text { provean algunos instrumentos básicos iniciales. }\end{array}$ \\
\hline \multicolumn{2}{|c|}{ Instrumentos de promoción } & $\begin{array}{l}\text { - } \quad \text { Talleres de planificación participativa. } \\
\text { - Análisis DAFO. } \\
\text { - Formación general en economía social, empresarial y técnica. } \\
\text { - Asesoría (animación grupal, primer análisis del mercado...). } \\
\text { - } \quad \text { Ensayo mediante precooperativas. } \\
\end{array}$ \\
\hline \multirow[b]{2}{*}{$\begin{array}{l}\text { Agentes de } \\
\text { promoción } \\
\text { (autopromoción } \\
\text { vs. promoción } \\
\text { externa) }\end{array}$} & Agentes internos & $\begin{array}{ll}\text { - } & \text { Futuros socios. } \\
\text { - } & \text { Primeros líderes internos. } \\
\end{array}$ \\
\hline & Agentes externos & $\begin{array}{l}\text { - Líderes locales externos. } \\
\text { - } \text { Movimiento de economía social nacional o internacional. } \\
\text { - Universidades. } \\
\text { - Sindicatos. } \\
\text { - Administración pública local, regional o nacional. }\end{array}$ \\
\hline
\end{tabular}

Fuente: elaboración propia a partir de Cornforth y Thomas (1990), Préfontaine y Léger (1990) y Brown y Baker (1989)

Para que una empresa de economía social nazca (sea creada) antes debe haber sido concebida y gestada. Las fases de concepción inadecuadas suelen abocar al posterior fracaso o a procesos plagados de dificultades, ya sea por exceso de intervención de terceros (cooperativas inducidas exógenamente para cubrir los objetivos de agentes ajenos a los propios socios) o por integración incompleta del grupo de emprendedores (socios demasiado heterogéneos o que no han madurado suficientemente sus ideas o sus planes colectivos). En este sentido, Vargas (1995) menciona el caso de muchas cooperativas constituidas desde el desempleo o por la crisis previa de empresas capitalistas, de forma obligada, sin un proceso que haya permitido la madurez y homogeneidad del grupo. De modo similar, Morales (1996) se refiere a las pseudocooperativas y a las cooperativas tayloristas; las primeras son las entidades inducidas por un agente externo para satisfacer sus propios intereses; el segundo término se refiere a los acuerdos entre el antiguo jefe y los trabajadores de una empresa para transformarla en una cooperativa como instrumento de reflotamiento. Estos fenómenos organizativos no están necesariamente condenados a la ausencia de participación o a la quiebra, pues la presencia de líderes representativos puede reconducirlos hacia acciones colectivas más o menos conscientes. En todo caso, la inercia de la experiencia inicial suele ser un lastre que condiciona el desarrollo futuro y dificulta el proceso de adquisición de competencias (Morales, 1998).

Teniendo claros estos peligros que acechan a cualesquiera empresas de participación, ¿cómo evitarlos en el caso particular de aquéllas promovidas en la universidad? Esto es, ¿cómo gestar, en las instituciones académicas superiores y desde un principio, grupos 
naturales de emprendedores? El epígrafe que sigue está basado, entre otros aspectos, en la experiencia de promoción del espíritu empresarial en diversas universidades españolas por los autores del presente artículo. Parte de estas experiencias y reflexiones se recogen en Coque, López y Loredo (2006) y Coque, Falagán, Fernández, García, López, Loredo y Novelle (2007). Para la redacción de este epígrafe se han tenido también en cuenta las referencias Valnalón (2000a, 2000b).

\subsection{Apoyo al emprendimiento desde la universidad}

El Espacio Europeo de Educación Superior (EESE) ha adoptado como reto el tránsito hacia un modelo de docencia más centrado en los procesos de aprendizaje de los alumnos, que facilite en mayor medida el desarrollo de competencias (Salinas, 2004). Frente a enfoques tradicionales de los programas de formación, centrados en la adquisición de los conocimientos, la pedagogía orientada hacia la adquisición de competencias no se basa en lo que el alumno deberá saber al concluir una materia o ciclo, sino en las acciones que tendrá que ser capaz de efectuar después de haber superado un periodo de aprendizaje (De Miguel, 2005). Con la nueva metodología que se pretende implantar, muchas de las capacidades valoradas por la sociedad, y que deberían tenerse en cuenta a la hora de reformar los planes docentes, se encuentran en un estrecho vinculo con la capacidad emprendedora: iniciativa y creatividad, información, relación y comunicación, resolución de problemas y toma de decisiones en ambientes de incertidumbre, gestión del tiempo, responsabilidad personal, etc. Parece muy probable que la capacidad emprendedora se vea favorecida con este cambio.

Por otro lado, en el Libro Verde sobre El espíritu empresarial en Europa (2003) se pone de manifiesto la necesidad de fomentar el dinamismo empresarial de un modo eficaz en la región y se articulan directrices para tratar de crear un entorno favorable para que las empresas se creen, se cierren, se adquieran, prosperen y sobrevivan. Una de las medidas contempladas es la educación y formación en el espíritu empresarial para el fomento de una actitud favorable y para desarrollar la sensibilización hacia salidas profesionales como empresario y las competencias correspondientes.

La puesta en marcha de una empresa exige empuje, creatividad y tenacidad, mientras que su desarrollo precisa cada vez más capacidad de gestión. Teniendo en cuenta que tanto la personalidad como las aptitudes de gestión son elementos claves del éxito, las competencias personales relacionadas con el espíritu empresarial deberían enseñarse desde una fase 
temprana y hasta el nivel universitario, en el que el interés podría centrarse en la formación de la capacidad de gestión. La Comisión Europea considera que la mayor parte de los Estados miembros se han comprometido, en diferentes grados, a promocionar la enseñanza del espíritu empresarial en sus sistemas educativos (Comisión de las Comunidades Europeas, 2003).

Muchos países desarrollan formación en este sentido ya desde los primeros ciclos de enseñanza. Por ejemplo, en España, varias Comunidades autónomas participan en la implantación y desarrollo de la asignatura Empresa Joven Europea. En Asturias, donde se observa una experiencia pionera en este campo liderada desde hace una veintena de años por el centro de empresas Valnalón, que ha sido exportada a otras regiones españolas y a otros países, se trata de una asignatura optativa oficial dentro del sistema educativo de la región.

Esta asignatura está dirigida a jóvenes de 15 a 18 años, que deben gestionar durante el curso una cooperativa de comercio internacional en la que intercambian productos con cooperativas de alumnos de otros países a través de videoconferencias. Luego, venden en el mercado local los productos importados. Los países con los que se coopera son tanto europeos (Portugal, Francia, Alemania, Reino Unido, etc.) como pertenecientes a otros continentes (Canadá, EE.UU., Méjico, Ecuador, Colombia, etc.). El éxito del programa hace que cada año el número de alumnos y cooperativas participantes sea mayor, y el proyecto haya sido considerado como buena práctica según la Unión Europea en el año 2006. El programa para el periodo 2004-2007, se renovó para el periodo 2008-2011, dentro del Acuerdo para la Competitividad, el Empleo y el Bienestar de Asturias (ACEBA).

En suma, el nuevo planteamiento docente a nivel europeo fomenta capacidades muy valoradas en el campo del emprendimiento. Existen directrices de fomento del espíritu emprendedor que incluyen la recomendación de distintas medidas. Entre ellas se encuentra el fomento de la capacidad y las competencias emprendedoras, introduciendo la educación y formación en todos los niveles educativos.

Las universidades están en una posición privilegiada para llevar a cabo un fuerte fomento de la cultura emprendedora entre su alumnado, personal docente y de investigación. Así, se considera muy importante la incorporación a los planes de estudio de asignaturas centradas en esta materia, así como todo tipo de formación no reglada, ya sean charlas, pequeños cursos, seminarios, etc. En el caso de España, la Red de Oficinas de Transferencia de Resultados de Investigación (OTRI ) tiene el objetivo de dinamizar las relaciones entre el 
mundo científico universitario y el de la empresa y sirve de punto de información y asesoramiento para nuevos emprendedores. Además, se observa que en los últimos años algunas universidades crean sus propios órganos de motivación, formación y semilleros empresariales, con el objetivo de impulsar con más fuerza la aparición de emprendedores universitarios.

Un ejemplo claro puede encontrarse en el Programa IDEAS, de la Universidad Politécnica de Valencia que, desde el año 2001 en que fue creado, ha promovido la creación de más de 360 empresas. Más de 36 universidades dispondrían de programas de creación de empresas, pero no existe una única fórmula, probablemente por encontrarse aún en un estado de evolución en el que cada entidad académica busca un camino propio con base en su saber hacer (Millet, 2008).

En la Universidad de Oviedo se ha creado recientemente, dentro del Área de Empleabilidad, un programa específico de apoyo al emprendimiento con distintas actividades: concurso de ideas empresariales, formación específica a través de cursos de verano sobre cómo elaborar un plan de empresas y charlas de motivación con la colaboración de la Asociación Jóvenes Empresarios de Asturias. Asimismo, como en muchas otras universidades, la OTRI es un organismo que también tiene encomendadas algunas de estas tareas. Sus tres misiones son: la transferencia de tecnología y conocimiento, la difusión de cultura científica, y la motivación y el desarrollo empresarial. Sin embargo, se detecta una dispersión y un uso ineficiente de recursos al comprobar que también otras unidades organizativas de la misma universidad, como el Instituto Universitario de Tecnología Industrial de Asturias (IUTA), incluyen entre sus objetivos el fomento del emprendimiento. La OTRI organiza charlas, seminarios y jornadas, atiende a los emprendedores que se acercan a sus instalaciones, permite utilizar alguno de los puestos de trabajo de que dispone, etc., todo ello restringido por unos recursos humanos y materiales muy limitados, por lo que en gran parte su misión se apoya en la colaboración con los Centros de Empresas del área de influencia de cada campus de esta universidad: Valnalón (posteriormente sustituido por el CEEI) en el Campus de Mieres, el Centro Municipal de Empresas de Gijón, en el Campus de Gijón y el CEEI, en los Campus de Oviedo.

Si bien es destacable la cooperación con los Centros de Empresas externos a la universidad, no se puede obviar el hecho de que escasean los medios destinados a esta misión 
dentro de la propia institución, especialmente al observar otras universidades españolas, como la de Santiago de Compostela, con una sociedad de Capital Riesgo propia, o el desarrollado programa de semillero de la Universidad Politécnica de Valencia, ya mencionado más arriba (Valnalón, 2000a).

Al igual que ocurre con la implantación de políticas de calidad, la introducción del espíritu emprendedor en la universidad precisa de un fuerte liderazgo estratégico y de un enfoque ambicioso, que no tiene porqué estar reñido con una puesta en marcha pragmática. En caso contrario, se corre el riesgo de que prevalezca la actual visión de corto plazo, con actuaciones descoordinadas, discontinuas, insuficientemente conectadas con el entorno extraacadémico y condicionadas al voluntarismo de unos pocos o a los recursos financieros que se puedan ir consiguiendo. En este sentido, Rodeiro et al. (2010) han encontrado que tanto los recursos financieros destinados a investigación básica y aplicada como el número de personas trabajando en las OTRI influyen positivamente en la generación de spin-offs en la universidad española.

En un nivel más operativo, puede identificarse una serie de actuaciones relacionadas con el itinerario del emprendedor universitario que deben ser replanteadas:

- Puesta en marcha de viveros de empresas con la dotación de equipos y personal precisos.

- Implantación de una o varias asignaturas abiertas relacionadas con la creación de empresas en todos los campus.

- Incremento de la notoriedad interna y externa del emprendimiento universitario.

- Obtención de financiación especifica para mantener a un consultor senior en la universidad, al menos un día a la semana. Adicionalmente, este servicio podría ser impulsado mediante la firma de un convenio con alguna ONG como SECOT (Seniors Españoles para la Cooperación Técnica).

- Coordinación bajo un mismo organismo universitario de todas las actividades de fomento del emprendimiento realizadas dentro de la institución.

- Puesta en marcha de un foro de promoción de emprendedores, con el fin de vincular en todo el proceso a entidades externas a la universidad. 
- Intercambio de experiencias y la cooperación entre distintas universidades, como abono para un crecimiento generalizado de la cultura emprendedora y para la mejora de la eficacia de los recursos destinados a este fin.

En suma, existe la necesidad de que las universidades tomen la iniciativa de promover y fomentar la cultura emprendedora y dediquen recursos a ello, en proporción a la importancia que esta cuestión tiene, desde el nivel educativo hasta la puesta en marcha de un Centro de Empresas promovido por cada universidad. Por otra parte, allí donde se instale un Centro de Empresas debería buscarse la posibilidad de cooperar con la universidad de la zona; esto favorece la aparición de sinergias para beneficio mutuo (Lalkaka, 1997), sinergias que se traducen en la posibilidad de formar emprendedores, de vender tecnología, de crear empresas innovadoras surgidas de investigaciones en la universidad, etc. Es necesario aprovechar también las sinergias que se derivan de establecer redes de colaboración ínter universitarias en este campo. Finalmente, una visión de conjunto a la luz del modelo de intervención de Valnalón (2000a, 2000b) sugiere considerar a la universidad como un eslabón más de una cadena de emprendedores que comienza mucho antes y finaliza mucho después. Esto exige romper estructuras tradicionales universitarias, basadas en grupos de trabajo aislados, para cooperar mucho más, interna y externamente.

Parece claro que las acciones realizadas actualmente desde la universidad no son suficientes para promover la creación de empresas universitarias, pero ¿tienen algún efecto? ¿Son capaces de gestar grupos naturales de emprendedores impulsando alguno de los sucesos disparadores apuntados en el epígrafe anterior? ¿Se evitan los riesgos mencionados de exceso de intervención o integración incompleta de los socios? Para dar respuesta a estas preguntas se ha realizado un estudio empírico basado en empresas colectivas emprendidas por estudiantes de tres universidades españolas.

\section{ESTUDIO EMPÍRICO}

\subsection{Metodología}

Uno de los objetivos planteados en este artículo fue la realización de un estudio comparativo de casos que permitiera conocer la realidad de iniciativas empresariales colectivas llevadas a por estudiantes egresados de las universidades participantes en el

\footnotetext{
REVESCO No 112 - Extraordinario en Homenaje al Profesor Alfonso Carlos MORALES

GUTIÉRREZ - ISSN: 1885-8031 - www.ucm.es/info/revesco
} 
estudio. Estos estudios de casos se llevaron a cabo mediante una metodología de entrevistas en profundidad a socios de dichas empresas.

Previo a la realización de las entrevistas, hubo que determinar las variables del estudio. Estas variables se construyeron a partir de la revisión de la literatura científica. Tras el proceso de construcción del marco teórico, se marcaron cinco grandes áreas: definición conceptual; factores sociales; factores educativos; factores psicológicos; experiencias. La revisión bibliográfica de estas cinco áreas permitió configurar las variables de la entrevista cuyo guión reproduce la tabla 2.

Tabla 2. Guión de las entrevistas a emprendedores universitarios

\begin{tabular}{|c|c|}
\hline & $\begin{array}{l}\text { A. Datos de la empresa } \\
-\quad \text { Nombre de la empresa } \\
- \text { Fórmula jurídica } \\
- \text { Localidad } \\
- \text { Año de constitución } \\
-\quad \text { Principales actividades } \\
-\quad \text { N. }{ }^{\circ} \text { de socios trabajadores } \\
-\quad \text { N. de socios no trabajadores } \\
-\quad \text { N. }{ }^{\circ} \text { de trabajadores no socios } \\
\text { B. Datos sociodemográficos del socio } \\
-\quad \text { Titulación } \\
-\quad \text { Edad al crear la empresa } \\
-\quad \text { Sexo } \\
\text { C. Comentarios del entrevistador }\end{array}$ \\
\hline & $\begin{array}{l}\text { D. Trayectoria personal y laboral } \\
\text { - Describa brevemente a su familia ( } \mathrm{n}^{\text {o }} \text { de hermanos, ocupaciones y estudios de cada uno) } \\
\text { - Explique su trayectoria y formación/educación a nivel de primaria, secundaria y superior } \\
\text { - Explique su recorrido de actividades laborales (antes y después de sus estudios) } \\
\text { E. Motivos para crear la empresa } \\
\text { - Explique los principales motivos por los que decidió participar en la creación de esta empresa } \\
\text { - Por qué lo hizo junto con varias personas más (explique varios motivos) } \\
\text { - Explique cómo fue el proceso de formación del grupo de socios (¿qué tenían en común? ¿quién ha } \\
\text { entrado o salido posteriormente?) } \\
\text { F. Percepciones } \\
\text { - Características o atributos que debe tener un empresario para el éxito } \\
\text { - ¿A qué atribuye los éxitos y fracasos que ha tenido en su negocio? } \\
\text { - Principales problemas que encontraron al iniciar el negocio } \\
\text { - ¿Qué apoyos recibieron (universidad, familia, otros agentes) antes y durante el proceso de creación de } \\
\text { la empresa? } \\
\text { - Cuéntenos cómo ha evolucionado el proceso de toma de decisiones entre los socios de la empresa } \\
\text { (cómo lo hacían al principio y en la actualidad) }\end{array}$ \\
\hline
\end{tabular}

Fuente: elaboración propia

Sin embargo, aun definiendo dichas variables, dada la implicación de investigadores de tres universidades distintas se hizo necesario elaborar una serie de orientaciones que unificaran los criterios de aplicación de la entrevista, mejorando con ello la fiabilidad de la misma, todo ello sin perjuicio de que al tratarse de una herramienta de las denominadas 
cualitativas, dichas orientaciones no restringieran en modo alguno las respuestas de las personas entrevistadas. Uno de los primeros criterios a considerar fue la selección de las personas a entrevistar. En este caso, en las distintas reuniones mantenidas entre los miembros del equipo se acordó entrevistar a socios de empresas colectivas de participación (es decir, que en su gestión estuvieran participadas democráticamente por varios de sus socios independientemente de su fórmula jurídica). El número de socios trabajadores mínimos de la empresa debían ser tres y la empresa debía ser de reciente creación, considerando por tanto que no tuviese una antigüedad superior a cinco años. Cada una de dichas empresas debía tener además un carácter universitario, o sea integrada por socios con titulación universitaria y una actividad comercial afín a la misma (por ejemplo, una piscifactoría gestionada por biólogos).

Sobre el método de muestreo concreto, éste se hizo de tipo incidental.

Las empresas fueron contactadas previamente, concertando un día y una hora para llevar a cabo las entrevistas. En dicho contacto inicial se les explicó el motivo de la investigación aunque sin ser demasiado preciso con el fin de no contaminar futuras respuestas. Todas las entrevistas se hicieron personalmente en la sede social de la empresa con el fin de obtener mayor información verbal y no verbal de los entrevistados. Las entrevistas fueron grabadas (tras solicitar la autorización de los entrevistados) para su posterior trascripción y análisis. Además, el entrevistador tomaba pocas notas manuscritas durante la entrevista para poder centrarse en el proceso.

Para asegurar la fiabilidad y validez del instrumento mediante una aplicación homogénea, se puso especial énfasis en leer las preguntas como estaban en el guión, evitando así que el entrevistador introdujera sesgos. Dichas preguntas se formularon de una manera lo bastante amplia para no inducir determinadas respuestas.

Las entrevistas se realizaron entre los meses de julio y agosto de 2008. Se llevaron a cabo entrevistas a empresarios universitarios de trece empresas colectivas (tabla 3). 
Tabla 3. Empresas participantes en el estudio de casos y sectores de actividad

\begin{tabular}{|l|l|}
\hline Nombre de la empresa & Actividad \\
\hline -Aceites Atenea & -Envasado y comercialización de aceite \\
-AST Ingeniería & -Simulación de sistemas mecánicos y de control \\
-Bionova Calidad & -Seguridad y calidad alimentaria \\
-Dicontec Automatización & -Sistemas de control y automatización \\
-Ecología y Calidad Consultores & -Gestión ambiental de la empresa \\
-Grontal Soluciones Biológicas & - Fabricación de material de laboratorio y comercialización \\
-Ingeniería, Electricidad y Energía Solar & -Instalación de energías renovables \\
-Innova Sistemas de Gestión e Innovación & -Estudios geotécnicos y ambientales \\
-Melocotón Creativo & -Diseño creativo y publicitario de moda \\
-Mitra Ingeniería Sostenible & -Energías renovables y consultoría \\
-Poniente Prevención & -Servicios de prevención de riesgos laborales y formación \\
-RS Creativa & -Consultoría y asesoría en responsabilidad social corporativa \\
-Ruralízate & -Central de reservas de turismo rural \\
\hline
\end{tabular}

Fuente: elaboración propia

Recogida la información, el siguiente paso fue la trascripción de todas las entrevistas así como al análisis y codificación de las mismas. La etapa de análisis y codificación es uno de los momentos críticos en la investigación cualitativa ya que una de las principales dificultades es la interpretación de los datos. La recogida de amplia información con un cierto nivel de ambigüedad conlleva un mayor nivel de complejidad que el análisis estadístico de datos cuantitativos (Yin, 2009). Esta dificultad es aún mayor en el caso de una investigación como la presente, donde han participado equipos de diferentes universidades. La codificación es el proceso en el que las respuestas largas se reducen y clasifican en categorías específicas de respuestas (Sommer y Sommer, 1997); lógicamente, las respuestas categóricas (sexo, edad, profesión...) tienen fácil codificación; sin embargo, éste se complica en el caso de respuestas más complejas.

De manera genérica, existen dos formas de codificar no necesariamente excluyentes (Strauss y Corbin, 1998): mediante códigos abiertos, es decir haciendo un primer acercamiento de la información recogida, señalando palabras o trozos de texto que llamen la atención al investigador; mediante codificación axial, es decir, generando categorías y subcategorías así como las relaciones entre sí, intentando buscar una explicación (en este caso se utilizan diagramas o representaciones gráficas de las categorías así como de las relaciones entre ellas).

En este estudio se utilizaron ambos sistemas de codificación. Así, en un primer momento se hizo una lectura de la trascripción, señalando con un marcador aquellas palabras o trozos de discurso interesantes o importantes. 
Esta primera lectura se completó con una audición posterior de la grabación, para poder captar adecuadamente la intencionalidad, ritmo y entonación del entrevistado. También en esta audición se comprobó si había alguna interacción del entrevistador que hubiera podido condicionar o viciar la respuesta. Debemos pensar que en el proceso de entrevista, las respuestas se desarrollan en un contexto social de interacción entrevistador-entrevistado que lo condiciona enormemente.

Tras esta primera lectura y audición, se realizó una segunda. En ella se fueron anotando al margen de la hoja, las distintas categorías y subcategorías del discurso. Esta categorización de las respuestas permitió, de una parte establecer las relaciones entre ellas y de otra destacar las categorías más importantes o sensibilizadoras para la persona entrevistada. Dichas categorías y sus relaciones fueron posteriormente representadas en una hoja aparte obteniendo un mapa cognitivo. Un ejemplo del mapa cognitivo de una de las entrevistas lo podemos ver en el gráfico 1. Esta representación reflejaba no sólo las distintas categorías descubiertas sino su importancias con respecto a las otras así como las relaciones entre ellas (Ruiz-Olabuénaga, 1999). Para esta segunda lectura se tuvieron en cuenta no solo las trascripciones de las entrevistas, sino también las notas recogidas por el entrevistador que aportan elementos valorativos, tales como la entonación o las expresiones faciales, muy importantes en el proceso de análisis. 
Gráfico 1. Ejemplo de mapa cognitivo

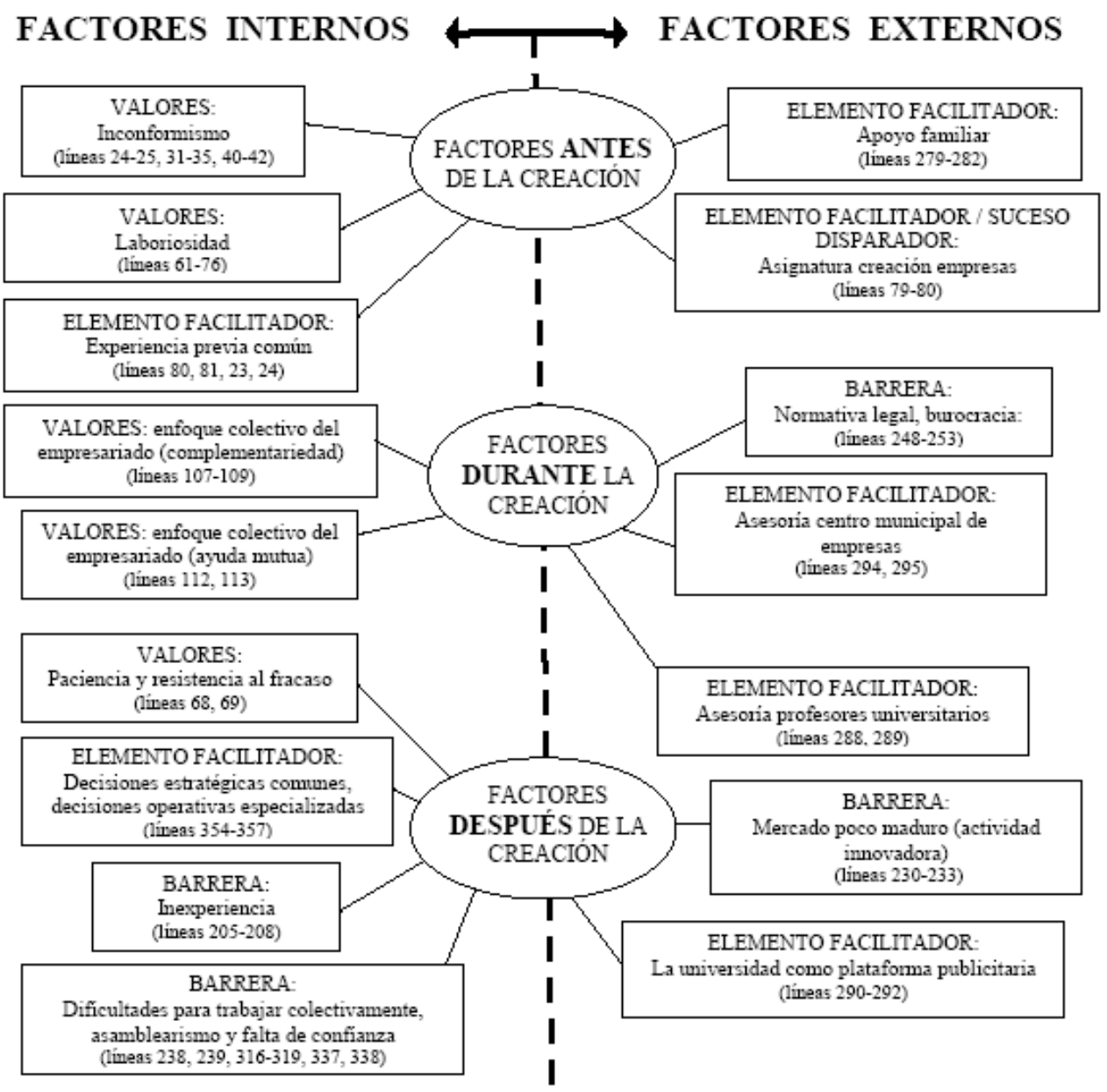

Fuente: elaboración propia

Un elemento importante a tener en cuenta en el análisis es la consistencia interna de cada entrevista individual, de manera que ésta será buena si no se producen cambios de puntos de vista durante la misma. Deberán anularse las entrevistas con baja consistencia interna, ya que pueden distorsionar al resto de las entrevistas analizadas.

En resumen, al final de todo este proceso se elaboraron dos documentos por cada una de las entrevistas: Por una parte, frases o párrafos señalados en la primera lectura. De otra, un mapa de categorías, señalando su importancia (utilizando tamaño de letra o colores distintos) y sus relaciones (mediante flechas u otros conectores). 


\subsection{Resultados}

A continuación se resumen las variables que, según las entrevistas realizadas, serían las principales facilitadoras del proceso de creación de una empresa participativa. Manteniendo el guión trazado en la fundamentación teórica, en primer lugar se reflejan las respuestas relacionadas con la creación del grupo de emprendedores y de la propia empresa (los antecedentes que suponen experiencias laborales tempranas, los motivos para crear la empresa y la conformación del grupo), para seguidamente dejar constancia de la promoción externa recibida (el apoyo familiar, el apoyo desde la universidad).

\subsubsection{Experiencias laborales tempranas}

Una de las características en la mayoría de las entrevistas, ha sido el hecho de que los empresarios empezaron a iniciarse en el mercado laboral en edades muy tempranas, a la vez que cursaban sus estudios. Esta decisión, a veces tenía la finalidad de financiar los estudios, en otras ocasiones no. Se observa por tanto una inquietud hacia el mundo laboral que incita a estos emprendedores a empezar a trabajar en diversos oficios.

He trabajado durante la carrera en el Corte Inglés y en pequeños trabajillos, y cuando terminé la carrera, pues, desde que la terminé, en oficinas de farmacia yyyy, aquí y después en verano también me fui, un verano, me fui a Mallorca a trabajar yyy, y ya la empresa (Empresaria, 28 años).

Sí, de todo, camarero, he estado en una empresa de limpieza, he hecho de todo. Cuidé a una anciana, también (Empresario, 35 años).

Bueno, además de ser dependienta en Cortefiel, mientras estaba acabando la carrera, después a través de las prácticas estuve llevando la publicidad de una marca de aquí de Granada que se llama "Darvec" y después de eso ya formé la empresa con las otras socias (Empresaria, 28 años).

Durante la carrera estuve de prácticas y en Ávilas Rojas (Empresario, 32 años).

También, durante la carrera, durante todo estos años, durante toda mi vida (Empresario, 24 años). 


\subsubsection{Motivos para crear la empresa}

En cuanto a los motivos expresados para tomar la decisión de crear la empresa, pueden agruparse, tras el análisis de las entrevistas, en dos grandes categorías: desarrollo profesional $\mathrm{y}$ valores personales.

Muchas de las respuestas a la pregunta sobre los motivos por los que decidieron crear la empresa giran en torno a su desarrollo profesional y su deseo de trabajar en aquello que les gusta y que no pueden encontrar en el trabajo por cuenta ajena:

Cuando estás trabajando en empresas, bueno, te puede gustar más o menos, pero es тиy difícil que el cargo, el puesto, la empresa, todo lo que desempeñes sea lo que te gusta a ti (Empresario, 28 años).

Trabajar en un laboratorio o en cualquier otra rama es muy complicado, normalmente trabajas como técnico, no trabajas como licenciado y aparte es muy difícil entrar, eeehhh, probé la parte de la nutrición con el Máster yyyy, tampoco me convenció, me gusta, pero tampoco me convenció yyyy, estuve haciendo prácticas en hospitales y eso, pero no me veía yo en eso (Empresaria, 28 años).

En cualquier caso, este hecho se relacionará directamente con los valores y actitudes de los emprendedores, ya que frente a esa insatisfacción hacia los puestos que ofrece el mercado laboral, subyacen diversos valores personales que estimulan la búsqueda de otras alternativas, entre ellas el autoempleo. Entre estos valores, uno de los que ha sido expresado en diversas entrevistas, ha sido el grado de iniciativa, el deseo de innovar, de crear y probar nuevas experiencias:

Esa habilidad es lo que yo creo que distingue a una persona trabajadora a otra que sea empresario. Ese gusto por la iniciativa (Empresaria, 28 años).

Motivación, me faltaba motivación, llevaba ya muchos años trabajando en lo mismo (Empresario, 35 años).

Esta iniciativa se relaciona también con el deseo de libertad e independencia que algunos entrevistados han manifestado: 
No tienes jefe, entonces puedes hacer lo que te dé la gana, pero sobre ti cae lo que hagas (Empresaria, 28 años).

El no depender de, de ningún jefe, el ser yo mi propio jefe y, y ser responsable de mis actuaciones (Empresario, 32 años).

Otro de los valores predominantes, ha sido la capacidad de adaptación:

Pues sobre todo, uuhm, facilidad para resolver lo que te pueda ir surgiendo por el camino (Empresaria, 28 años).

Tener capacidad de adaptación y capacidad de trabajo y de no importarte, que lo mismo un día trabajas ocho horas que otro día trabajas doce, o trabajas los fines de semana (Empresaria, 28 años)

(Sobre los motivos de fracaso) Pues a, muchas veces a la cabezonería, de que, por aquí tenemos que ir, por aquí tenemos que ir y por aquí tenemos que ir (Empresaria, 28 años)

Valor asimismo expresado como necesario para crear la empresa ha sido la constancia:

Yo creo que sobre todo la constancia, la constancia y el trabajar mucho, porque como sea una persona que se aburre en seguida no llega a nada (Empresario, 24 años).

Sin saber mucho de lo que significaba una empresa colectiva muchos se vieron atraídos por las ideas democráticas que conlleva, el poder formar una empresa de todos, y de modo innovador plantear una empresa.

Cuando se les preguntó directamente a los entrevistados sobre las cualidades o atributos que debe tener un empresario para llevar a su empresa al éxito la que más es reconocida es el ser perseverante y comprometido. Junto con ello el liderazgo, no entendido como una jefatura sino como una capacidad de generar motivación y confianza a compañeros en la empresa y a clientes de ésta, planteando metas y expectativas objetivas, y junto con ello la creatividad y el orden, son características a las que ampliamente aspiran a poder llegar a cumplir los entrevistados tras su propia experiencia cooperativa. Estas cualidades entonces 
están directamente relacionadas a la hora de referirse sobre las causas de los éxitos o fracasos de las empresas.

\subsubsection{Composición del grupo}

Como ya se ha indicado uno de los criterios tenidos en cuenta al seleccionar las empresas es que éstas estuvieran constituidas por varios socios. Aquí, interesaba conocer tanto los motivos que tuvieron en cuenta para crear la empresa junto con otros socios, como el proceso de elección de los socios como la toma de decisiones.

Con respecto a los motivos para decidir crear una empresa en grupo, de las entrevistas recogidas se ha podido observar cómo en la mayoría de los casos se trata de buscar equipos complementarios, en las funciones y tareas, por lo que es frecuente encontrar empresas integradas por personas de distintas titulaciones de procedencia, o bien emprendedores que poseen la misma titulación pero que en la empresa se han especializado en alguna área concreta.

La idea surgió un poco entre todos y, cada uno tenía un, cada uno tenía, cada una tenemos una parte que aportar a la empresa que para nosotros es muy importante ¿no? Que no, las dos no somos especialistas en lo mismo, una es más comercial, la otras es más administrativa, una tiene unos conocimientos, otra tiene otros, una es más positiva, otra es más negativa, entonces, no sé, empezamos así y nos compensamos, y en realidad vemos que, que somos complementarias, no, si una falta se nota (Empresaria, 28 años).

Yo tenía una parte muy específica de un trabajo, entonces necesitaba sobre todo la parte de gestión que de eso yo no había estudiado nada y la parte de diseño gráfico que mi educación iba más por el campo artístico. Entonces esos dos tipos de estudios que han hecho ellas me complementaban a mí y yo a ellas, claro, entonces así decidimos formar la empresa (Empresaria, 28 años).

Cada uno éramos buenos en una, o sea, veníamos de haber trabajado en distintas empresas, y cada uno sabíamos más de una parte (Empresario, 28 años).

REVESCO No 112 - Extraordinario en Homenaje al Profesor Alfonso Carlos MORALES 
Además de esta complementariedad, existe afinidad personal entre los miembros del equipo que hace que la atracción y el compromiso hacia el proyecto sean mayores:

Teníamos una forma de ver la vida bastante parecida, los cuatro somos de pueblo, Alhama, Montefrío, y también queríamos trabajar en nuestro pueblo, entonces eso sí, sí ha servido de engranaje para después consolidarnos como grupo (Empresario, 32 años).

Hablas con unos, hablas con otros y encuentras a otra persona que te ayuda a pegar el empujón a, a crear tu propia empresa, era un amigo mío, un vecino, él estudió económicas (Empresario, 35 años).

Un aspecto interesante con relación al grupo es el relativo a la toma de decisiones. Al ser empresas colectivas, este punto es verdaderamente interesante, ya que es una de las claves principales en la supervivencia y mantenimiento de la empresa. Pues bien, del análisis de las respuestas se observa que en la mayoría de los casos la toma de decisiones se hace delegando parcelas de responsabilidad a cada socio:

Al principio, tomábamos las decisiones consensuadamente, digamos, entre los tres, ¿no?, porque somos administradores solidarios, los tres, pero luego vimos que eso no era la forma idónea porque se bloqueaban las decisiones bastante [...] hace relativamente poco tiempo decidimos cada uno tener, ser responsable, de un área, como dividir la empresa en compartimentos más o menos estancos, y que cada uno sea responsable de uno o dos áreas [...] así pues ya la cosa es más fluida (Empresario, 28 años).

Al principio todos hacemos de todo porque no hay trabajo [...] después pues te van dividiendo, pues, por áreas, pues administración, técnico, comercial, y ya pues o cada socio o quien se contrate se encarga de tomas las decisiones en ese tema (Empresario, 32 años).

En cualquier caso, los empresarios reconocen que la toma de decisiones es uno de los problemas más difíciles al que se enfrentan:

Primero que nada, a un empresario social está claro que no es el lucro lo que lo mueve, a diferencia de la empresa de corte tradicional, ¿verdad? Por lo 
tanto es mucho más difícil, donde tienes que convivir con otros, porque ya es asociativa, porque es una empresa más de personas, donde importa el otro, donde importa la decisión. Todos sabemos que no es fácil una organización democrática, es lo más difícil ejercer la democracia, la toma de decisiones democrática. En cambio el empresario privado es dueño de sus acciones, y en la medida que tiene más acciones es que decide. Por lo tanto, es bastante más fácil (Empresaria, 25 años).

De todas maneras, la comunicación es una herramienta de gestión que estas empresas utilizan a través de distintos métodos, como las reuniones semanales:

Nosotras desde el principio hablamos mucho y tenemos reuniones periódicas, haya o no haya un problema. La comunicación creemos que es importante y saber, aunque cada una lleve una parte, un departamento, saber que está haciendo la otra... tampoco es tener un control, pero sí una visión de cómo se va desarrollando el trabajo (Empresaria, 28 años).

\subsubsection{Apoyo familiar}

Uno de los principales problemas que señalaron los empresarios a la hora de iniciar el negocio era la dificultad para capitalizar la empresa y acceder al crédito. Así, en la mayoría de los casos será la familia quien juegue un papel muy importante de soporte financiero en los primeros momentos de la constitución de la empresa:

Tú tienes que tener un apoyo económico muy fuerte que normalmente la gente se lo apoya sus padres (Empresario, 35 años).

Nosotros no teníamos ni un duro ninguno, excepto yo que dejé mi trabajo y cobré el desempleo en pago único los demás tuvieron que pedir el dinero en su casa, no era mucho, pero la empresa necesitaba comprar una serie de instrumental (Empresario, 32 años).

Pero este apoyo, no sólo es en términos financieros, sino que en algunos casos es también moral y de aprendizaje mediante modelo. Este hecho es especialmente interesante por la identificación de género en el caso de los padres e hijos varones: 
Mi padre tiene una empresa de distribución de material de laboratorio y mi madre era ama de casa (Empresario, 35 años).

La ocupación de mis, no tengo madre, y mi padre es, eh, autónomo, constructor, se dedica a la construcción (Empresario, 32 años).

\subsubsection{Apoyo desde la Universidad}

Un aspecto interesante en el análisis de las entrevistas a los emprendedores es que éstos, a pesar de simultanear estudios y trabajo, una vez titulados siguen dando mucha importancia a la formación continua de manera que siguen estudiando y formándose a la vez que han creado la empresa, especialmente en temas de gestión de empresas:

En el año 2002 hice prácticas mientras estudiaba [...] en realidad ahora también estoy estudiando (Empresario, 28 años).

Mmmm, ganas de trabajar, un buen proyecto, luchar por ese proyecto y después una formación continua tanto, tanto en lo que se va a dedicar esa empresa, como en temas de administración y dirección de empresas (Empresario, 32 años).

La formación que falta universitaria en todas las ramas, no sólo gente de económicas y empresariales montan negocios, y es necesario, ya te digo, tanto para los que están por cuenta ajena, como para los que estamos por cuenta propia (Empresaria, 28 años).

Pero, mucho antes en el ciclo del emprendimiento colectivo, varias de las personas entrevistadas valoran como elemento facilitador previo el haberse podido conocer en asignaturas universitarias de creación de empresas, donde además desarrollaron una versión previa del actual proyecto empresarial:

Entonces fue más pensando en que seguíamos haciendo el trabajo de clase aunque fuese fuera de la universidad [...] Surgió así en el trabajo de clase, empresas, electrónica, un prototipo, estaba obligado a hacerlo con esos compañeros porque era con los que estaba en clase en ese momento y con los únicos que podía hacerlo. Surgió bien, ganamos el premio al que nos 
presentamos, con el que se fomentaba este trabajo de clase, y la idea surgió en la cafetería preparando un trabajo que fuese lo más atractivo posible (Empresario, 25 años).

[...] la afinidad de las personas con las que empecé, porque el proyecto lo empecé haciendo un proyecto en una asignatura en la carrera, en Creación de Empresas, conocí a un grupo amplio de gente, primero éramos ocho personas creo, no con las ocho me llevé igual, pero hubo dos de ellas, una ya era amiga mía y otro lo conocí allí que es mi actual socio, y con ellos dos, empezamos a pensar la idea de hacerlo seriamente (Empresaria, 25 años).

Además, la universidad ha facilitado a varias de las personas entrevistadas otros servicios útiles como asesoría, plataforma publicitaria, apoyo anímico o contactos profesionales e institucionales:

Consultamos con un montón de gente, profesores, gente que se implicó además personalmente fuera de lo que es estrictamente tal, o sea que estamos muy contentos también, y después de haberla puesto, pues [...] dando unas charlas, es como echar nosotros una mano a la universidad, pero en realidad es al revés (Empresario, 27 años).

Contactos [...] y la parte de la universidad que trató con nosotros y que es de donde salimos [...] los profesores del departamento de empresas también hablaron con nosotros y demás, los profesores que tuvieron que ver con ese proyecto, toda la gente que estuvo alrededor de ese concurso, de ese proyecto de clase, toda esa gente fue por parte de la universidad de la que recibimos ese apoyo, y nos presentó gente del ayuntamiento, y luego nos ayudó también, nos aconsejó, y el ánimo (Empresario, 25 años).

\section{CONCLUSIONES Y PROPUESTAS}

En resumen, de las entrevistas realizadas podemos señalar como variables facilitadoras en el proceso de creación de empresa las siguientes:

- Inicio en la actividad laboral a edades tempranas. 
- Valores como iniciativa, independencia o adaptación.

- Desarrollo profesional.

- Identificación con otros socios (estudios, localidad).

- Complementariedad en la elección de socios.

- Dificultad inicial en la toma de decisiones.

- Apoyo familiar en aspectos financieros o antecedentes empresariales.

- Apoyo desde la universidad mediante asignaturas de creación de empresas, asesoría, contactos, plataforma publicitaria o formación continua.

Esta última fuente de apoyos se percibe muy secundaria respecto a la anterior, de lo que se deduce que queda aún mucho que mejorar en el ámbito académico. En ese sentido, los párrafos que siguen, basados en la experiencia profesional de los autores del presente artículo, desarrollan algunas propuestas en este ámbito.

Una medida frecuente para ir creando espíritu emprendedor en el medio académico son las charlas informativas sobre la posibilidad de crear una empresa al finalizar los estudios. Normalmente son dirigidas al alumnado de los últimos años de cualesquiera carreras, aunque sería interesante que comenzaran a darse antes, desde los cursos iniciales, con el fin de facilitar la generación tranquila y reflexionada de futuros grupos de emprendedores una vez obtengan sus títulos varios años después. Otro colectivo que debería asistir a estas acciones de difusión son los investigadores senior y los becarios de proyectos de investigación, para sembrar en ellos la inquietud de crear spin-off universitarias. Todas estas charlas pueden ser impartidas por personal de un servicio específico dotado por cada universidad, que deberá ser apoyado por expertos ajenos a la institución y por emprendedores jóvenes que ya hayan puesto en marcha sus empresas. El método, muy sencillo, encierra una dificultad importante: cómo atraer asistentes cuando la población de los campus universitarios es generalmente reacia a acudir a conferencias que no reporten beneficios inmediatos aparentes.

Una solución es combinar o completar las conferencias de libre asistencia con otras actividades más prolongadas y participativas que cuenten con público cautivo: asignaturas, normalmente optativas, a lo largo de las cuales el alumnado debe organizarse por grupos y 
desarrollar un estudio de mercado semiprofesional y un plan de negocio realista sobre ideas propuestas por ellos mismos basadas en los conocimientos específicos que les aporta la carrera que están estudiando. Estas materias, permiten el aprendizaje cooperativo por prueba y error y fomentan en una parte del alumnado el impulso de crear una empresa real con sus compañeros, muchas veces similar a la simulada en el aula. Es importante que, además del profesorado responsable de estas asignaturas, colaboren de nuevo asesores externos a la universidad procedentes de instituciones públicas locales, de ONG especializadas, de asociaciones empresariales o del movimiento cooperativo, quienes participarán en algunas de las sesiones de seguimiento de los proyectos. A partir de la experiencia más frecuente, limitada a estudios de gestión o técnicos, debe extenderse esta oferta formativa a cualesquiera títulos de cada universidad, e incrementar sus contenidos a aspectos como la innovación o la gestión de proyectos. Debe aplicarse, asimismo, una combinación de herramientas tradicionales (simulación de realidades empresariales dentro y fuera del aula) con otras más innovadoras (tele trabajo colaborativo mediante TIC), evitando el peligro fácil de centrarse en las segundas, lo que dificulta la interacción directa, necesaria para la generación de grupos de emprendedores. Otra medida útil es la vinculación de estas asignaturas con otras de la misma titulación, de modo que el alumnado trabaje menos en cada una de ellas y adquiera una visión transversal de sus estudios. Además, aunque la mayor parte de experiencias limitan la formación de los grupos a estudiantes de una misma titulación, sería interesante la puesta en marcha de medidas que faciliten la creación de empresas multidisciplinares.

La actividad de las asignaturas de creación de empresas puede ser hecha pública mediante jornadas celebradas a fin de curso con presencia en los medios de comunicación y de asistencia abierta a autoridades académicas, a agentes del entorno local, a otros estudiantes y miembros de la comunidad universitaria y al público en general. Las jornadas se centrarán en la exposición y discusión de los proyectos empresariales elaborados por el alumnado, pero incluirán también intervenciones de emprendedores reales jóvenes -con quienes los asistentes se sientan fácilmente identificados- y un concurso de ideas que genere un clima festivo de competitividad sana entre los proyectos presentados. Quienes ganen ese concurso deberán ser estimulados para que reelaboren posteriormente sus planes de negocio con el apoyo de asesores profesionales -pertenecientes o no a la universidad- con el fin de estudiar la posibilidad de ponerlos realmente en marcha en un futuro próximo. 
Más en general, deben estimularse, ponerse en marcha y articularse entre sí otros muchos recursos de apoyo: investigación multidisciplinar aplicada al fomento del emprendimiento, apoyo a las relaciones entre universidades en este campo, desarrollo de herramientas formativas específicas (modelos de plan de empresa universitarios adaptados a diferentes titulaciones, programas informáticos de apoyo...), puesta en marcha y actualización frecuente de bases de datos y de observatorios tecnológicos útiles para la generación de ideas empresariales y para los estudios de mercado, financiación para concursos de ideas, servicio de asesoría, servicio de consulta de estudios de mercado o planes de negocio ya realizados... La gestación de grupos de emprendedores exige que la formación y asesoría ofrecida no se limite sólo a los aspectos empresariales y técnicos, sino que también deberá trabajarse con el ámbito de la dinámica de grupos y la ideología cooperativa, desarrollando el proyecto empresarial a partir del interés y las capacidades de cada miembro; hay que discutir con detalle los pros y los contras de emprender colectivamente; además, deberá estimularse y moderarse la figura de los líderes, muy importantes en la fase inicial de cada grupo. De entre todas estas acciones, las más especializadas no pueden dejarse en manos de becarios o personal joven con escasa experiencia como se suele hacer, sino que exigirán profesionales con largo recorrido en la consultoría o la creación de sus propias empresas, que podrán ser reclutados en el entorno local ajeno al medio académico.

La visibilidad de todas estas acciones precisa que se disponga de espacios específicos localizados en sitios céntricos o de paso, donde grupos de emprendedores potenciales podrán trabajar junto a empresas que se hallen en fases posteriores de su ciclo de vida. Estos semilleros compartidos por la pre-incubación y la incubación facilitan el aprendizaje mutuo y la articulación de futuras alianzas estratégicas.

La importancia de la coordinación es clave para la totalidad de estas medidas que, con demasiada frecuencia, son impulsadas por grupos reducidos de profesorado con más voluntad que recursos, y cuyas responsabilidades institucionales suelen encontrarse dispersas entre diferentes servicios, unidades organizativas y vicerrectorados. Es evidente que la atomización de acciones reduce su eficacia, eficiencia y visibilidad, lo cual es especialmente grave cuando de lo que se trata es justamente de lo contrario: de crear un caldo de cultivo donde, de forma natural e integrada, vayan generándose futuros grupos de emprendedores en diferentes estadios de su ciclo que interactúen y aprendan, mutuamente y con otros agentes de apoyo pertenecientes o no al medio académico. Como se indicaba al final del epígrafe anterior, la 
gestación del emprendimiento colectivo en la universidad debe verse como una fase más de un ciclo que comienza en los estudios primarios y secundarios, y continúa en el mundo empresarial extra universitario, con los cuales hay que establecer acuerdos formales de colaboración. Es muy importante, en particular, la relación con los centros de empresas.

Además, para aumentar el profesorado implicado hay que motivarlo mediante contraprestaciones que reconozcan el esfuerzo que exige colaborar en estas actividades. Posibles medidas pueden ser la exención de docencia o el reconocimiento académico formal al mismo nivel que los méritos de docencia o investigación.

Es necesario, en suma, que la dirección de las instituciones académicas asuman como suya la filosofía que subyace en este artículo y que establezcan formal y explícitamente los medios para su realización práctica en forma de discursos públicos, partidas presupuestarias, servicios de apoyo y coordinación dotados de personal experimentado, y convenios de colaboración con entidades externas pertenecientes al ámbito público, empresarial, financiero, asociativo o de la economía social.

\section{BIBLIOGRAFÍA}

BROWN, J.; BAKER, L. (1989). Co-operative Training \& Development. London: London Co-operative Training.

COMISIÓN DE LAS COMUNIDADES EUROPEAS (2003). Libro verde El espíritu empresarial en Europa. Bruselas: Comisión de las Comunidades Europeas.

COQUE, J.; FALAGÁN, B.; FERNÁNDEZ, B.; GARCÍA, S., LÓPEZ, N.; LOREDO, E.; NOVELLE, J. (2007). Fomento del espíritu emprendedor en la Universidad de Oviedo en colaboración con el Ayuntamiento de Gijón. Gijón: Ayuntamiento de Gijón.

COQUE, J.; LÓPEZ, N.; LOREDO, E. J. (2006). Fomento de la cultura emprendedora e innovadora entre el alumnado universitario del campus politécnico de Gijón. Forum Calidad, 177, 42-50.

CORNFORTH, C.; THOMAS, A. (1990). Cooperative Development: Barriers, Support Structures and Cultural Factors. Economic and Industrial Democracy, 11, 451-461.

DE MIGUEL, M. (2005). Cambio de paradigma metodológico en la Educación Superior. Exigencias que conlleva. Cuadernos de Integración Europea, 2, 16-27. 
ENTRIALGO, M.; FERNÁNDEZ, E.; VÁZQUEZ, C.J. (2001). El comportamiento emprendedor y el éxito de la PYME: modelos de contingencia y configuraciones. Dirección y Organización, 25, 47-58.

GARCÍA-GUTIÉRREZ, C. (1991). La economía social o la economía de las empresas de participación (las sociedades cooperativas y laborales). En: VV.AA., En memoria de María Angeles Gil Luezas. Madrid: Alfa Centauro, 195-216.

HUNT, G.C. (1992). Division of Labour, Life Cycle and Democracy in Worker Co-operatives. Economic and Industrial Democracy, 13, 9-43.

KELMAR, J.H.; WINGHAM, L.W. (1995). Determining the Relevant Factors in the Success Strategies of Small Enterprises. The Journal of Entrepreneurship, 4(2), 215-236.

LALKAKA, R. (1997). Lessons from international experience for the promotion of business incubation systems in emerging economies. Viena: United Nations Industrial Development Organization.

MILLET, J. (2008). La Universidad crea empresa: claves de las spin-off. Ponencia Día del Emprendedor, Mieres.

MORALES, A.C. (1998). Competencias y valores en las empresas de trabajo asociado. Valencia: CIRIEC-España.

MORALES, A.C. (1996). Ineficiencia del mercado y eficacia de las cooperativas. Valencia: CIRIEC-España.

PRÉFONTAINE, J.; LÉGER, L. (1981). Los problemas financieros durante la evolución de las cooperativas. En: M. LAFLAMME (Ed.), Gestión moderna de cooperativas. Santafé de Bogotá: Fondo Nacional Universitario, 197-225, 1990. Versión en español de: La gestion moderne des coopératives, Montreal: Gaëtan Morin.

RAFFA, M.; ZOLLO, G.; CAPONI, R. (1996). The Development Process of Small Firms. Entrepreneurship and Regional Development, 8(4), 359-371.

RODEIRO, P.; FERNÁNDEZ, S.; OTERO, L.; RODRÍGUEZ, A. (2010). Factores determinantes de la creación de spin-offs universitarias. Revista Europea de Dirección y Economía de la Empresa, 19 (1), 47-78.

RUIZ-OLABUÉNAGA, J. I. (1999). Metodología de la investigación cualitativa. Bilbao: Deusto.

RUTHERFORD, M.W.; OSWALD, S.L. (2000). Antecedents of Small Business Perfomance. New England Journald of Entrepreneurship, 3(2), 21-33. 
SALINAS, J. (2004). Innovación docente y uso de las TIC en la enseñanza universitaria. Revista Universidad y Sociedad del Conocimiento, 1, noviembre.

SINGH, R.P.; HYBELS, R.C.; HILLS, G.E. (2000). Examining the Role of Social Network Size and Structural Holes. New England Journal of Entrepreneurship, 3(2), 47-58.

SOMMER, B. y SOMMER, R. (1997). Practical Guide to Behavioural Research. Nueva York: Oxford.

STRAUSS, A. y CORBIN, J. (1998). Basics of Qualitative Research. Techniques and Procedures for Developing Grounded Theory. Londres: Sage Publications.

TRUSEN, C. (1994). Descentralización y participación: el rol de las organizaciones de autoayuda. En: VV.AA., Memorias del Seminario-Taller Internacional El Desarrollo Rural en América Latina hacia el Siglo XXI. Tomo I. Santafé de Bogotá: Pontificia Universidad Javeriana, 165-180.

VALNALÓN (2000a). Cadena de formación de emprendedores. Memoria 1987-2006. Langreo: Ciudad Industrial del Valle del Nalón.

VALNALÓN (2000b). Detección, apoyo y formación de emprendedores universitarios. Langreo: Ciudad Industrial del Valle del Nalón.

VARGAS, A. (1995). La identidad cooperativa y la cooperativa como empresa: luces y sombras. Revista de Estudios Cooperativos (REVESCO), 61, 179-192.

VECIANA, J.M. (1999). Creación de empresas como programa de investigación científica. Revista Europea de Dirección y Economía de la Empresa, 8(3), 11-36.

YIN, R.K. (2009). Case Study Research: Design and Methods. Fourth Edition. California: SAGE Publications. 